\title{
Equivalence theorem of $D$-optimal equal allocation design for multiresponse mixture experiments
}

\author{
Xiaoyuan Zhu (D), Honghua Hao (D), Weixia Li (D), Chongqi Zhang*(D) \\ School of Economics and Statistics, Guangzhou University, Guangzhou, 510006, P.R. China
}

\begin{abstract}
The equivalence theorem is the most important theorem of experimental design. For single response, the D-optimal equivalence theorem of the continuous design and equal allocation design already exist. However, the equivalence theorem of D-optimal equal allocation design for multiresponse mixture experiments has not been investigated. In this paper, we study this problem and find that the maximize of the variance function of the equivalence theorem equal to the number of response. D-optimal designs for multiresponse are illustrated by two examples.
\end{abstract}

Mathematics Subject Classification (2020). 62K05, 62K20

Keywords. Multiresponse, mixture experiment, D-optimal, equivalence theorem, equal allocation

\section{Introduction}

In experimental design, according to the research sequence, the single response model was first studied by researchers. Multiresponse experiments have a wide range of applications in many fields, such as biology, medicine, economics, engineering, environmental science, and clinical medicine. Therefore, when the study of single response is sufficient, it is time to examine multiresponse. Multiresponse experiments involve two or more responses with one model, and there may be correlations between these responses. This paper considers the multiresponse of mixture experiment models. There are $q$ ingredients in the mixture experiment. The explanatory variables are the $q$ ingredients proportions $x_{1}, x_{2}, \ldots, x_{q}$. The key feature of these explanatory variables is that they sum to 1 . We can denote the experiment region as

$$
S^{q-1}=\left\{\left(x_{1}, x_{2}, \ldots, x_{q}\right): \sum_{i=1}^{q} x_{i}=1, x_{i} \geq 0, i=1,2, \ldots, q\right\} .
$$

The design of an experiment is an essential part of the statistical methodology to improve scientific experimentation and thus, increase the validity of the resulting conclusions [27]. The experimental design of the multiresponse model was first studied by Draper and

\footnotetext{
* Corresponding Author.

Email addresses: ailydiaai@gmail.com (X.Y. Zhu), huahua19850217@163.com (H.H. Hao), liweixia851019@163.com (W.X. Li), cqzhang@gzhu.edu.cn (C.Q. Zhang)

Received: 06.08.2020; Accepted: 15.04.2021
} 
Hunter [7]. Wijesinha [34] investigated experimental designs with known and unknown correlations between responses. Krafft and Schaefer [19] and Chang [4] studied some properties of multiresponse. Imhof [12] and Chang et al. [5] also studied some examples of multiresponse. Liu and Yue [23] developed some multiresponse theorems. Liu and Yue [24] and Liu et al. [25] studied multiresponse linear mixed models. Two textbooks have focused on the design of multiresponse experiments [1,14]. Wong et al. [35] studied optimal design for multiresponse nonlinear regression models. This paper mainly considers multiresponse models for mixture experiments. Next, we introduce some references about multiresponse in mixture experiments. Smith and Cornell [32] studied biplot displays for visualizing multiple response data. Jin and Yue [13] studied the D- and A-optimal designs for mixture experiments with multiresponse models. Mandal and Pal [26] used a weight combination design and studied the A-optimal design of the multiresponse mixture K-model. Pal and Mandal [28] studied the D- and A-optimal design of the two responses of the mixture model. A textbook on the design of mixture experiments [6] mentioned multiresponse.

Equivalence theorem is the fundamental theorem of D- and other optimal design criteria. The D-optimal design is defined formally by Kiefer and Wolfowitz [18] and is the most commonly used criterion. The D-optimal design criterion is proposed based on the parameter estimation. For a given design, the equivalence theorem seeks to prove whether the design is the optimal design. However, according to the equivalence theorem, there is not a strict method to find the optimal design. Therefore, there are many papers about the optimal design for specific models. For example, Laake [20] gave a classic solution for optimal design. Atwood [2] investigated an easy method to verify the D-optimal design through geometric properties. The G-optimal design was defined formally by Kiefer and Wolfowitz [18]. The G-optimal design criterion is proposed based on the response estimation. The equivalence theorem posits that these two design criteria are identical when the design is expressed as a measure of experiment region. Kiefer and Wolfowitz [17] investigated the equivalence theorem of D-optimal design, Kiefer [15] explored a more general equivalence theorem for optimal criteria, and Spruill and Studden [33] extended the equivalence theorem to random processes. Ceranka and Graczyk [3], Hassanein and Kilany [11] used the equivalence theorem for the optimal design of experiments. Li and Zhang [21], Li and Zhang [22], and Zhu et al. [37] used the equivalence theorem for the optimal design of mixture experiments.

Scheffé $[30,31]$ proposed the mixture experimental design's foundational work with the canonical polynomial model of the mixture experiment. Its simple lattice design and simplex centroid design are equal allocation designs and satisfy the D-optimal criterion for common mixture models. The quadratic mixture canonical polynomials with spline [36] research confirmed that D-optimal designs are equal allocation designs. More broadly speaking, many studies have considered the theoretical research on continuous design, and in practical application, they have to be changed into the exact design for implementation. The equal allocation design can significantly reduce the number of tests and cost. The number of tests at each design point is 1 , which is incredibly convenient for implementing the experiment. This paper investigates the equivalence theorem of D-optimal equal allocation design for multiresponse mixture experiments. The theoretical system is improved, and it can measure the excellence of medium allocation designs in practical applications.

In this paper, we mainly give the equivalent theorem of D- and G-optimal design and variance function for the multiresponse mixture model under the equal allocation experimental design. In Section 2, we present the necessary explanation and notations. We investigate the equivalence theorem of equal allocation for multiresponse mixture design in Section 3. In Section 4, we provide an example to illustrate the result. Finally, all technical details are given in the Appendix. 


\section{Model specification and preliminaries}

In single response, let $y_{i}=f^{\mathrm{T}}\left(x_{i}\right) \beta+\varepsilon_{i}(i=1, \ldots, N)$ be a general linear regression model on $S^{q-1}$, where $y_{i}$ is the response observed value, $f=\left(f_{1}, \ldots, f_{p}\right)^{\mathrm{T}}$ represents the vector of known regression functions, $x_{i}=\left(x_{i 1}, x_{i 2}, \ldots, x_{i q}\right) \in X \subset S^{q-1}, X$ is a compact set region in $S^{q-1}$, and $\beta$ is the unknown parameter vector, with $E\left(\varepsilon_{i}\right)=0$. Because the errors are independent, with constant variance, $E\left(\varepsilon_{i} \varepsilon_{l}\right)=0(i \neq l), E\left(\varepsilon_{i}^{2}\right)=\sigma^{2}$, and $N$ is the total experiment frequency.

The form of the multiresponse mixture model is

$$
y_{i u}=f_{u}^{\mathrm{T}}\left(x_{i}\right) \beta_{u}+\varepsilon_{i u}, \quad i=1, \ldots, N ; \quad u=i, \ldots, r .
$$

The $r$ responses for observation $i$ are correlated, but the observations $i$ and $l$ are independent $i \neq l$, with $E\left(\varepsilon_{i u}\right)=0, E\left(\varepsilon_{i u} \varepsilon_{i v}\right)=\sigma_{u v}$. Because the observations at different design points are independent, we have $E\left(\varepsilon_{i u} \varepsilon_{l u}\right)=E\left(\varepsilon_{i u} \varepsilon_{l v}\right)=0, u, v=1, \ldots, r$, where $y_{i u}$ is the $u$ th response value of the $i$ th observation, $f_{u}^{\mathrm{T}}$ is a set of known function vectors for the $u$ th response, $\beta_{u}$ is the column vector of unknown parameter for the $u$ th response, and $\varepsilon_{i u}$ is the error of the $u$ th response of the $i$ th observation. Manifestly, in matrix form, the model becomes

$$
Y=F(x) \beta+\varepsilon,
$$

where $Y=\left(y_{1}, y_{2}, \ldots, y_{r}\right)^{\mathrm{T}}$ is $r \times 1$ matrix, $F(x)=\operatorname{diag}\left(f_{1}^{\mathrm{T}}(x), f_{2}^{\mathrm{T}}(x), \ldots, f_{r}^{\mathrm{T}}(x)\right), \beta=$ $\left(\beta_{1}^{\mathrm{T}}, \beta_{2}^{\mathrm{T}}, \ldots, \beta_{r}^{\mathrm{T}}\right)$, and $\varepsilon=\left(\varepsilon_{1}^{\mathrm{T}}, \varepsilon_{2}^{\mathrm{T}}, \ldots, \varepsilon_{r}^{\mathrm{T}}\right)^{\mathrm{T}}$.

In Section 4, there are some simple examples to make it easier to observe the description of multiresponses. The variance-covariance matrix of the response (also known as error covariance matrix) is $\Sigma=\left\{\sigma_{u v}\right\}_{u, v=1, \ldots, r}$. Estimation of the parameter vector $\beta$ is by generalized least squares with weights $\Sigma^{-1}$. Error covariance matrix is an important index of multiresponse. Different papers have different settings and are not the same. Chang et al. [5] set $\Sigma=\left(\begin{array}{ll}1 & \rho \\ \rho & 1\end{array}\right)$. The literature [26,28] specified the values of the error covariance matrix as some commonly used values. However, it is generally similar to [13], set to $\Sigma=\left(\begin{array}{cc}\sigma_{1}^{2} & \rho \sigma_{1} \sigma_{2} \\ \rho \sigma_{1} \sigma_{2} & \sigma_{2}^{2}\end{array}\right)$.

Based on the model (2.1), an continuous design can be expressed as a probability distribution

$$
\xi=\left(\begin{array}{llll}
x_{1} & x_{2} & \ldots & x_{n} \\
\omega_{1} & \omega_{2} & \ldots & \omega_{n}
\end{array}\right)
$$

where $x_{i} \in X$ are finite support points and their weight $\xi\left(x_{i}\right)=\omega_{i}$ satisfy $\omega_{i}>0$ and $\Sigma \omega_{i}=1, \quad i=1,2, \ldots, n$. Let $\Xi$ be the class of all competing designs, and the $\Phi$-optimal design is to find the $\xi^{*} \in \Xi$ that can achieve the optimal design in some sense. The definition of the experiment plan is more in line with the actual experimental operation. Denote the equal allocation design as $\xi_{e}=\left(x_{1}, x_{2}, \ldots, x_{n}\right)$, where $x_{i} \in \mathcal{X}$, each $x_{i}$ is different from one an other, and run once at $x_{i}$. Let $\widetilde{\Xi}$ be the class of all equal allocation designs.

For all $r$ responses, the worth of a design is measured by its Fisher information matrix, which is given by Draper and Hunter [7]

$$
M(\xi, \Sigma)=\int_{x} f(x) \Sigma^{-1} f^{\mathrm{T}}(x) \xi(d x) .
$$

The variance function is

$$
\operatorname{Tr}\left(\Sigma^{-1} D(x, \xi, \Sigma)\right)=\operatorname{Tr}\left(\Sigma^{-1} f^{\mathrm{T}}(x) M^{-1}(\xi, \Sigma) f(x)\right) .
$$

The information matrix of single response is

$$
M(\xi)=\int_{x} f(x) f^{\mathrm{T}}(x) \xi(d x) .
$$


The variance function of single response is

$$
d(x, \xi)=f^{\mathrm{T}} M^{-1}(\xi) f(x) .
$$

Although the information matrix and variance function formula of single response and multiresponse are different, some notations are still represented by the same notations, except for the distinction between $d(x, \xi)$ and $D(x, \xi, \Sigma)$, because we can distinguish them according to the context.

Next, we introduce the D- and G-optimal criteria in the following equivalence theorem. For single response, Kiefer and Wolfowitz [17] showed that the following assertions are equivalent:

(S1): A design $\xi^{*}$ is D-optimal, ie., $\operatorname{det}\left(M\left(\xi^{*}\right)=\max _{\xi} \operatorname{det}(M(\xi))\right.$;

(S2): A design $\xi^{*}$ is G-optimal, ie., $\max _{\xi} d\left(x, \xi^{*}\right)=\min _{\xi} \max _{x} d(x, \xi)$;

(S3): $\max _{x} d\left(x, \xi^{*}\right)=k$, where $k$ is number of parameter;

(S4): $\max _{x} d\left(x, \xi_{e}^{*}\right)=1$.

Note that the information matrix in calculating the variance function is different in these assertions. The design $\xi^{*}$ is the continuous optimal design, whereas the design $\xi_{e}^{*}$ is the equal allocation optimal design.

Suppose $\Sigma$ and continuous measure $\xi$ as defined in equation (2.2). Federov [8] showed that the following assertions are equivalent:

(M1): A design $\xi^{*}$ is D-optimal, ie., $\operatorname{det}\left(M\left(\xi^{*}, \Sigma\right)\right)=\sup \operatorname{det}(M(\xi, \Sigma))$;

(M2): $\sup _{x} \operatorname{Tr}\left(\Sigma^{-1} D\left(x, \xi^{*}, \Sigma\right)\right)=\inf _{\xi}\left\{\sup _{x} \operatorname{Tr}\left(\Sigma^{-1} D\left(x, \xi^{*}, \Sigma\right)\right)\right\}$, where 'Tr' represents trace;

(M3): $\sup _{x} \operatorname{Tr}\left(\Sigma^{-1} D\left(x, \xi^{*}, \Sigma\right)\right)=k$, where $k$ is number of parameters.

The equivalence theorem plays an important role in proving that D-optimal design and G-optimal design are equivalent. It skillfully gave the upper limit of the variance function in assertion 3 of [17]. After the study of the equivalence theorem, we give the general design and the stochastic process. Research on multiresponse mixture has developed recently. When we study multiresponse mixture, we find an interesting theorem. It is shown in Section 3.

\section{Equivalence theorem of D-optimal designs}

In the single response, the equivalent theorem of D-optimal continuous design exists $[15,17]$, and the equivalent theorem of D-optimal equal allocation design also exists [15, 29]. In multiple responses, the equivalent theorem of D-optimal continuous design exists $[8,29]$. This section's theorem makes up for the equivalent theorem of multiresponse Doptimal design under equal allocation design. The theorem in this article will complete the conclusion of the equivalence theorem in various situations. It provides a basis for the verification of $\mathrm{D}$-optimal using equal allocation design under the multiresponse mixture model.

The simplex lattice design is the D-optimal design for the most commonly used secondorder canonical polynomial model. The simplex lattice design assigns equal weight to $C_{q}^{1}+C_{q}^{2}$ support points, which are the $C_{q}^{1}$ vertices of $S^{q-1}:(1,0, \ldots, 0), \ldots,(0, \ldots, 0,1)$, the binary mixtures in $S^{q-1}:(1 / 2,1 / 2,0, \ldots, 0), \ldots,(0, \ldots, 0,1 / 2,1 / 2)$. In the single response, assertion 4 of [17] shows that when the number of experiment runs for each support point is 1 , the calculation is more convenient. According to this, we infer that 
it has a similar theorem in multiresponse. There are two lemmas in this theorem, which involve the variance function and matrix determinant.

The following equivalence theorem for D-optimal design of the multiresponse mixture model is based on the equal allocation measure, which is simple and easy to implement. The equal allocation measure is an useful tool in the simplex lattice design with second- and third-order canonical polynomial mixture model; in the third-order canonical polynomial mixture model, Kiefer [16] gave 10 design points, but the design points differed from the third-order simplex lattice design with three components. The 10 design points are $(1,0,0),(0,1,0),(0,0,1),(b, 1-b, 0),(b, 0,1-b),(0, b, 1-b),(1-, b, 0),(0,1-b, b),(1-b, 0, b)$, and $(1 / 3,1 / 3,1 / 3)$, where $b=\left(1-5^{-1 / 2}\right) / 2$. Therefore, the design of the equal allocation measure can be widely used in multiresponse.

We can see that it assigns equal measure for each design point. It looks like it's just a design point. In other words, the abbreviation is as follows: $\xi_{e}=\left(x_{1}, x_{2}, \ldots, x_{n}\right)$. This still doesn't address the contradiction between $\Sigma \omega_{i}=1$ constraint and s constraint in Section 2.

The following two lemmas provide the necessary tools for the main results regarding the equivalence theorem of designs in multiresponse mixture models.

Lemma 3.1. Suppose $\xi_{e} \in \widetilde{\Xi}$, also $\operatorname{det}\left(M\left(\xi_{e}\right)\right) \neq 0$, then

$$
\int_{x} \operatorname{Tr}\left(\Sigma^{-1} D\left(x, \xi_{e}, \Sigma\right)\right) d \xi_{e}=r
$$

and

$$
\max _{x \in X} \operatorname{Tr}\left(\Sigma^{-1} D\left(x, \xi_{e}, \Sigma\right)\right) \geq r
$$

where $r$ is number of responses.

Proof. Because $\operatorname{Tr}\left(\Sigma^{-1} D\left(x, \xi_{e}, \Sigma\right)\right)=\operatorname{Tr}\left(\Sigma^{-1} F^{\mathrm{T}}(x) M^{-1}\left(\xi_{e}, \Sigma\right) F(x)\right)$, integrate the probability distribution on both sides of $\xi_{e}$, and we have

$$
\begin{aligned}
\int_{x} \operatorname{Tr}\left(\Sigma^{-1} D\left(x, \xi_{e}, \Sigma\right)\right) d \xi_{e} & =\int_{x} \operatorname{Tr}\left(\Sigma^{-1} F^{\mathrm{T}}(x) M^{-1}\left(\xi_{e}, \Sigma\right) F(x)\right) d \xi_{e} \\
& =\int_{x} \operatorname{Tr}\left(M^{-1}\left(\xi_{e}, \Sigma\right) F(x) \Sigma^{-1} F^{\mathrm{T}}(x)\right) d \xi_{e} \\
& =\operatorname{Tr}\left[M^{-1}\left(\xi_{e}, \Sigma\right) \int_{x} F(x) \Sigma^{-1} F^{\mathrm{T}}(x) d \xi_{e}\right] \\
& =\operatorname{Tr}\left[M^{-1}\left(\xi_{e}, \Sigma\right) M\left(\xi_{e}, \Sigma\right) d \xi_{e}\right] \\
& =\operatorname{Tr}\left(I_{r}\right)=r .
\end{aligned}
$$

Using the integral mean value theorem, the existence number $D^{*}$ satisfies:

$$
\int_{X} \operatorname{Tr}\left(\Sigma^{-1} D\left(x, \xi_{e}, \Sigma\right)\right) d \xi_{e}=\int_{X} D^{*} d \xi_{e}=D^{*}
$$

and

$$
\min _{x \in X} \operatorname{Tr}\left(\Sigma^{-1} D\left(x, \xi_{e}, \Sigma\right)\right) \leq D^{*} \leq \max _{x \in X} \operatorname{Tr}\left(\Sigma^{-1} D\left(x, \xi_{e}, \Sigma\right)\right) .
$$

Equation(3.1) shows $D^{*}=r$. From the above, formula (3.2) is proved.

Lemma 3.1 is used in the proof of Theorem 3.3, and Lemma 3.2 is used in the proof of Theorem 3.5.

Lemma 3.2. Let $\ln \left(\operatorname{det}\left(M\left(\xi_{e}\right)\right)\right)$ be a function on $M(\widetilde{\Xi})$, so this is a concave function. If $M\left(\xi_{e 1}\right) \neq M\left(\xi_{e 2}\right)$, we have

$$
\ln \left(\operatorname{det}\left(M\left(\xi_{e 1}\right)+M\left(\xi_{e 2}\right)\right)\right)>\ln \left(\operatorname{det}\left(M\left(\xi_{e 1}\right)\right)\right)+\ln \left(\operatorname{det}\left(M\left(\xi_{e 2}\right)\right)\right) .
$$


Proof. Because $M\left(\xi_{e 1}\right)$ and $M\left(\xi_{e 2}\right)$ is a positive definite matrix, let $0<\alpha<1$. Then, their determinants have the properties below

$$
\operatorname{det}\left(\alpha M\left(\xi_{e 1}\right)+(1-\alpha) M\left(\xi_{e 2}\right)\right) \geq\left[\operatorname{det}\left(M\left(\xi_{e 1}\right)\right)\right]^{\alpha}\left[\operatorname{det}\left(M\left(\xi_{e 2}\right)\right)\right]^{(1-\alpha)},
$$

where the condition that the equal sign is established is $M\left(\xi_{e 1}\right)=M\left(\xi_{e 2}\right)$, so,

$$
\operatorname{det}\left(M\left(\xi_{e 1}\right)+M\left(\xi_{e 2}\right)\right)>\left[\operatorname{det}\left(M\left(\xi_{e 1}\right)\right)\right]\left[\operatorname{det}\left(M\left(\xi_{e 2}\right)\right)\right],
$$

thus, (3.3) is proved.

The following two theorems are the main conclusions of this paper. Theorem 3.3 is about the equivalence of the D- and G-optimal design and the theorem of judging the optimal design according to the variance function. Theorem 3.5 is about the equality of the information matrix class and the design class that satisfy the three conditions in Theorem 3.3.

Theorem 3.3. Suppose the number of experiment runs at each design point is 1. In multiresponse mixture models, the following three conclusions are equivalent:

(i): A design $\xi_{e}^{*}$ is D-optimal, ie., $\operatorname{det}\left(M\left(\xi_{e}^{*}, \Sigma\right)\right)=\sup \operatorname{det}\left(M\left(\xi_{e}, \Sigma\right)\right)$;

(ii): A design $\xi_{e}^{*}$ is G-optimal, ie., $\operatorname{Tr}\left(\Sigma^{-1} D\left(x, \xi_{e}^{*}, \Sigma\right)\right)=\inf _{\xi_{e}}\left\{\sup _{x} \operatorname{Tr}\left(\Sigma^{-1} D\left(x, \xi_{e}, \Sigma\right)\right)\right\}$;

(iii): $\sup _{x} \operatorname{Tr}\left(\Sigma^{-1} D\left(x, \xi_{e}^{*}, \Sigma\right)\right)=r$, where $r$ is number of response.

The proof of Theorem 3.3 is given in the Appendix. Using Theorem 3.3, we obtained the following corollaries, which are generally called decision formulas.

Corollary 3.4. If the designs are all experiments with one test number, in the multiresponse mixture model, D-optimal design satisfies the following formula

$$
\phi(x)=\sup _{x} \operatorname{Tr}\left(\Sigma^{-1} D\left(x, \xi_{e}^{*}, \Sigma\right)\right)-r \leq 0 .
$$

Moreover, the supremum is achieved at all support points of $\xi_{e}^{*}$.

The information matrix is the whole representation of the experimental design and test plan. Therefore, we give some important theorems about the information matrix class and design class.

Theorem 3.5. Let three classes of information matrixes satisfy (i), (ii), (iii) in Theorem 3.3; then they are the same as each other. The linear combination of designs satisfying (i), (ii), (iii) of Theorem 3.3 also satisfies the condition (i), (ii), (iii) of theorem.

The proof of Theorem 3.5 is given in the Appendix. Next, we present two examples to illustrate Theorem 3.3 and Theorem 3.5.

\section{Examples for illustration}

To compare the efficiency of designs, define the D-efficiency of the design. Eff $_{D}=$ $\left(\frac{\operatorname{det}(M(\xi))}{\operatorname{det}\left(M\left(\xi_{D}\right)\right)}\right)^{1 / p}$, where $\xi_{D}$ is D-optimal design and $p$ is number of parameters. The maximum value of the variance function of D-optimal design is a certain constant. In addition, in the iterative algorithm of D-optimal design, a precision delta is added to the maximum value of variance function to stop iteration after sufficient demand, Li and Zhang [21] also applied this method. For this feature, we can consider the effect of an approximate optimal design, compare the design, and select this way.

Consider the following two examples, both of which are two-response models. Let the two responses be $Y_{1}$ and $Y_{2}$ with means $\eta_{1}(x)$ and $\eta_{2}(x)$. Obviously, $y_{i}=\eta_{i}(x)+\varepsilon_{i},(i=$ $1,2),\left(\begin{array}{cc}\sigma_{1}^{2} & \rho \sigma_{1} \sigma_{2} \\ \rho \sigma_{1} \sigma_{2} & \sigma_{2}^{2}\end{array}\right)$ is an error covariance matrix. 
Example 4.1. Suppose $\eta_{1}(x)$ is quadratic in $x$ whereas $\eta_{2}(x)$ is linear in $x$, and are given as

$$
\begin{gathered}
\eta_{1}(x)=\sum_{i=1}^{q} \beta_{i} x_{i}+\sum_{1 \leq i<j}^{q} \beta_{i j} x_{i} x_{j} \\
\eta_{2}(x)=\sum_{i=1}^{q} \alpha_{i} x_{i} .
\end{gathered}
$$

So, we can write

$$
E\left(\begin{array}{c}
Y_{1} \\
Y_{2}
\end{array}\right)=\left[\begin{array}{ccc}
f_{1}^{\mathrm{T}}(x) & 0_{q} & f_{2}^{\mathrm{T}}(x) \\
0_{q} & f_{1}^{\mathrm{T}}(x) & 0_{C_{q}^{2}}^{2}
\end{array}\right] \theta,
$$

where $f_{1}(x)=\left(x_{1}, x_{2}, \ldots, x_{q}\right)^{\mathrm{T}}, f_{2}(x)=\left(x_{1} x_{2}, x_{1} x_{3}, \ldots, x_{1} x_{q}, \ldots, x_{q-1} x_{q}\right)^{\mathrm{T}}, 0_{p}$ is a $1 \times p$ vector with all elements zero, and $\theta=\left(\beta_{1}, \ldots, \beta_{q}, \alpha_{1}, \ldots, \alpha_{q}, \beta_{12}, \beta_{13}, \ldots, \beta_{1 q}, . ., \beta_{q-1, q}\right)^{\mathrm{T}}$.

Jin and Yue [13] calculated the optimal continuous allocation on the second-order simplex lattice points. The advantage of this result is that it is independent of $\Sigma$. The weight of the support point $x \leftrightarrow(1,0, \cdots, 0)$ is $\frac{1}{q}-\frac{(q-1)\left(4+3 q-\sqrt{32+q^{2}}\right)}{2 q\left(q^{2}+3 q-2\right)}$, and the weight of the support point $x \leftrightarrow(1 / 2,1 / 2,0, \cdots, 0)$ is $\frac{4+3 q-\sqrt{32+q^{2}}}{q\left(q^{2}+3 q-2\right)}$, where $x \leftrightarrow(1 / 2,1 / 2,0, \cdots, 0)$. This means that two elements in $1 \times q$-dimensional vector are $1 / 2$ and the rest are 0 . Mandal and Pal [26] also studied this model; Theorem 2.3 shows that the barycenters of the factor space are the possible support point of the D-optimal design; Example 2.1 shows the D-optimal design with $q=3$ : the weight of the support point $x \leftrightarrow(1,0,0)$ is 0.1903 , and the weight of the support point $x \leftrightarrow(1 / 2,1 / 2,0)$ is 0.14307 .

We can use the method similar to [9] to set a large number of grid points on the definition domain when $q=2,3$ to verify. The verification result is that this design is D-optimal design and satisfies the equivalence theorem, and $q=4,5$ is the optimal continuous allocation.

Using the above conclusions, consider the performance of the simplex lattice design; at this time, any support point has equal weight. The following Table 1 compares the efficiency of the simplex lattice design with the optimal design. We consider simplex lattice design and D-optimal design when $q=2,3,4,5$, where $i$ is a degree of a vertex, $N_{i}=C_{q}^{i}$, The weights $\omega_{i}$ and the determinant of the information matrix $M$ of the two types designs are calculated. Then the efficiency $\mathrm{Eff}_{D}$ is obtained according to the determinant of the information matrix. Figure 1 describes the determinant and D-efficiency comparison results of the information matrix of the two designs. For simplicity of calculation, suppose

\begin{tabular}{|c|c|c|c|c|c|c|c|}
\hline \multicolumn{3}{|c|}{ Design } & \multicolumn{2}{|r|}{ Simplex lattice } & \multicolumn{2}{|r|}{ D-optimal } & Efficiencies \\
\hline Ingredients & $\bar{i}$ & 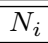 & $\overline{\overline{\omega_{i}}}$ & $\overline{\operatorname{det}(M(\xi))}$ & $\overline{\overline{\omega_{i}}}$ & $\overline{\operatorname{det}\left(M\left(\xi^{*}\right)\right)}$ & $\mathrm{Eff}_{D}$ \\
\hline$q=2$ & $\begin{array}{l}1 \\
2\end{array}$ & $\begin{array}{l}2 \\
1\end{array}$ & $\begin{array}{l}1 / 3 \\
1 / 3\end{array}$ & $3.85802 \times 10^{-4}\left(a b-c^{2}\right)^{2} b$ & $\begin{array}{c}0.375 \\
0.25\end{array}$ & $4.11987 \times 10^{-4}\left(a b-c^{2}\right)^{2} b$ & 0.98695 \\
\hline$q=3$ & $\begin{array}{l}1 \\
2\end{array}$ & $\begin{array}{l}3 \\
3\end{array}$ & $\begin{array}{l}1 / 6 \\
1 / 6\end{array}$ & $7.57057 \times 10^{-11}\left(a b-c^{2}\right)^{3} b^{3}$ & $\begin{array}{c}0.1903 \\
0.14307\end{array}$ & $8.42033 \times 10^{-11}\left(a b-c^{2}\right)^{3} b^{3}$ & 0.98825 \\
\hline$q=4$ & $\begin{array}{l}1 \\
2\end{array}$ & $\begin{array}{l}4 \\
6\end{array}$ & $\begin{array}{l}1 / 10 \\
1 / 10\end{array}$ & $5.02914 \times 10^{-21}\left(a b-c^{2}\right)^{4} b^{6}$ & $\begin{array}{l}0.11916 \\
0.08723\end{array}$ & $5.70673 \times 10^{-21}\left(a b-c^{2}\right)^{4} b^{6}$ & 0.99101 \\
\hline$q=5$ & & & $\begin{array}{l}1 / 15 \\
1 / 15\end{array}$ & $7.69571 \times 10^{-35}\left(a b-c^{2}\right)^{5} b^{10}$ & $\begin{array}{l}0.07947 \\
0.06026\end{array}$ & $8.80092 \times 10^{-35}\left(a b-c^{2}\right)^{5} b^{10}$ & 0.99331 \\
\hline
\end{tabular}
$\Sigma^{-1}=\left(\begin{array}{cc}a & c \\ c & b\end{array}\right)$

Table 1. D-efficiency of simplex lattice design (Example 4.1) 


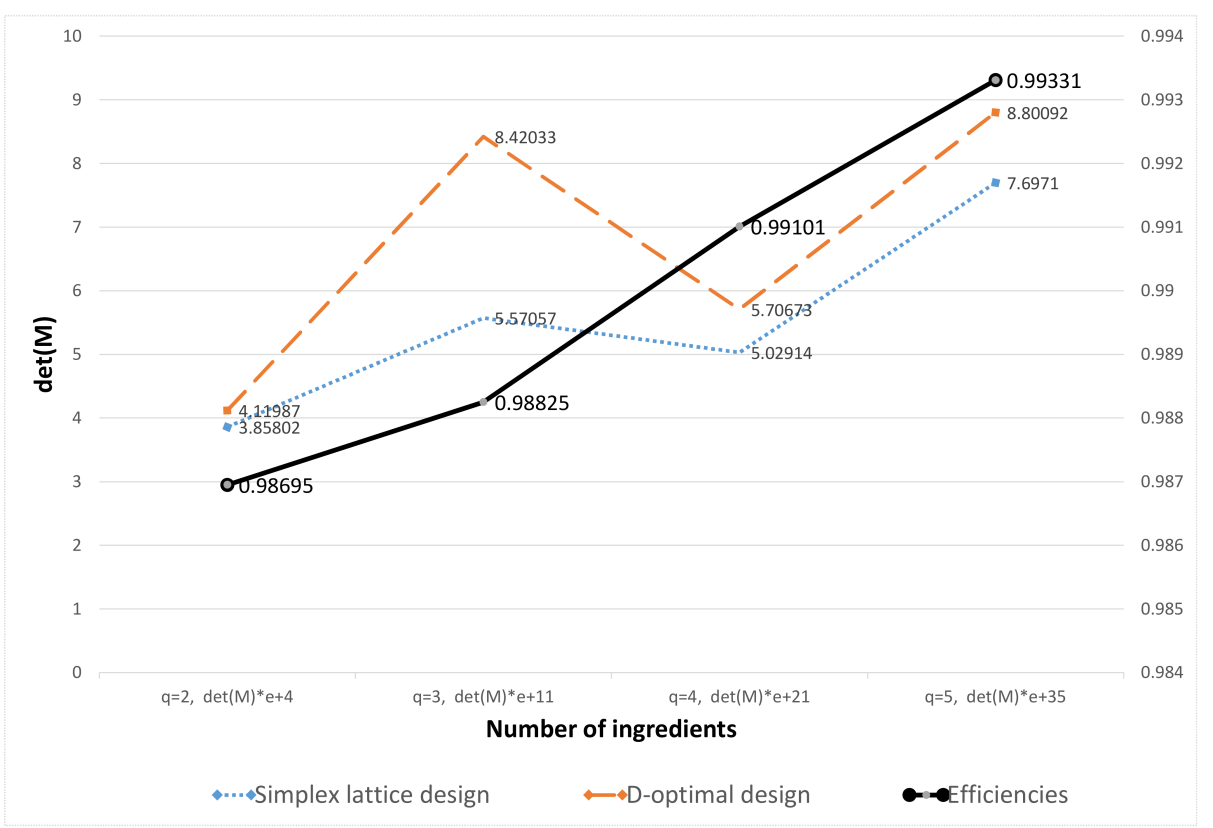

Figure 1. $\operatorname{det}(M)$ and D-efficiency of simplex lattice design (Example 4.1)

The D-efficiency of isometric simplex lattice design with 2,3,4,5 components is more than $98 \%$. The results show that the simplex lattice design with equal weights without calculating the optimal continuous allocation still has a good effect. Therefore, it is an excellent option to choose an equal allocation design. The following Example 4.2 is to verify Theorem 3.3 through the equal allocation design.

Using the two-response mixture model, which is composed of the seconder-order canonical polynomial with arbitrary finite-dimensional variables, Hao and Zhang [10] found that the D-optimal design of this model has an equal measure to each design point and independent of $\Sigma$.

Example 4.2. Suppose $\eta_{1}(x)$ and $\eta_{2}(x)$ is quadratic in $x$, and are given as

$$
\begin{aligned}
& \eta_{1}(x)=\sum_{i=1}^{q} \beta_{i} x_{i}+\sum_{1 \leq i<j}^{q} \beta_{i j} x_{i} x_{j}, \\
& \eta_{2}(x)=\sum_{i=1}^{q} \alpha_{i} x_{i}+\sum_{1 \leq i<j}^{q} \alpha_{i j} x_{i} x_{j} .
\end{aligned}
$$

So, we can write

$$
E\left(\begin{array}{c}
Y_{1} \\
Y_{2}
\end{array}\right)=\left[\begin{array}{cc}
f_{1}^{\mathrm{T}}(x) & 0_{q+C_{q}^{2}} \\
0_{q+C_{q}^{2}} & f_{2}^{\mathrm{T}}(x)
\end{array}\right] \theta
$$

where $f_{1}(x)=f_{2}(x)=\left(x_{1} x_{2}, x_{1} x_{3}, \ldots, x_{1} x_{q}, \ldots, x_{q-1} x_{q}\right)^{\mathrm{T}}$, and $0_{p}$ is a $1 \times p$ vector with all elements zero, and $\theta=\left(\beta_{1}, \beta_{2}, \ldots, \beta_{q}, \beta_{12}, \beta_{13}, \ldots, \beta_{1 q}, \ldots, \beta_{q-1, q}, \alpha_{1}, \alpha_{2}, \ldots, \alpha_{q}\right.$, $\left.\alpha_{12}, \alpha_{13}, \ldots, \alpha_{1 q}, \ldots, \alpha_{q-1, q}\right)^{\mathrm{T}}$.

Using the Lagrange multiplier method, we can find that D-optimal design has an equal measure for each design point as follows $\xi_{e}=(x \leftrightarrow(1,0, \ldots, 0), x \leftrightarrow(1 / 2,1 / 2,0, \ldots, 0))$.

To verify conclusion (iii) of Theorem 3.3 when $q=3$, we have the following information matrix

$$
M\left(\xi^{*}, \Sigma\right)=\frac{1}{4}\left(\begin{array}{cc}
a & c \\
c & b
\end{array}\right) \bigotimes\left(\begin{array}{cc}
M_{1} & \frac{1}{2} M_{2} \\
\frac{1}{2} M_{2} & \frac{1}{4} I
\end{array}\right)
$$


where $M_{1}=\left(\begin{array}{lll}6 & 1 & 1 \\ 1 & 6 & 1 \\ 1 & 1 & 6\end{array}\right), M_{2}=\left(\begin{array}{lll}1 & 1 & 0 \\ 1 & 0 & 1 \\ 0 & 1 & 1\end{array}\right), I$ is identity matrix and $\otimes$ is Kronecker product of matrix. The correlation matrix is

$$
M^{-1}\left(\xi^{*}, \Sigma\right)=\frac{1}{a b-c^{2}}\left(\begin{array}{ll}
b & c \\
c & a
\end{array}\right) \bigotimes\left(\begin{array}{cc}
I & -2 M_{2} \\
-2 M_{2} & 4 M_{1}
\end{array}\right) .
$$

The variance function is

$$
\operatorname{Tr}\left(M^{-1}\left(\xi^{*}, \Sigma\right) F(x) \Sigma^{-1} F^{\mathrm{T}}(x)\right)=2\left[\sum_{i=1}^{3} y_{i}^{2}+24 \sum_{i<j} y_{i}^{2} y_{j}^{2}+8 y_{1} y_{2} y_{3}-4 \sum_{i=1}^{3} y_{i}^{2}\left(1-y_{i}\right)\right] .
$$

According to [2], it can be verified that the variance function value at the vertex and the binary mixture is 2 , and at the center point is $34 / 27<2$, thus verifying conclusion (iii) in Theorem 3.3. Similarly, we can prove that the variance function is less than or equal to $r$ in $r$ responses.

\section{Further research}

Multiresponse is becoming suitable for social needs. For example, regional economic development requires fast development and high-quality and sustainable growth. Drug developemnt considers the ability to treat and the duration of the effect and drug resistance. Therefore, this paper's multiresponse mixture model is adaptable, the equal allocation design is easy to implement, and the D-optimal equivalence theorem is evaluative. Even for already implemented equal allocation designs, optimality can be compared and tested through the variance function.

In the future, we can study the following problems. Many multiresponse model experimental design papers have studied a lot of models; some are related to $\Sigma$, and some are not related to $\Sigma$. Another paper [24] introduced that multiresponse is sometimes unrelated to $\Sigma$ and sometimes related, and when related, the change of measurement with $\Sigma$ is small. The problem worthy of study is the relationship between the optimal design of multiresponse models and $\Sigma$. What is known now is that when each response model is the same, it has nothing to do with $\Sigma$, and when the response model is different, it is uncertain.

Many papers about multiresponse have considered the optimal design with all responses of each design point. But in reality, it is not necessary to observe all responses or the cost of some responses. Therefore, researchers can try to increase the number of trials or select some responses. In the optimal design of mixture experiments, we find an interesting problem: The single-saturation medium-saturation design holds that the number of design points is equal to the number of parameters, whereas the number of design points in a multiresponse design may be less than the number of parameters. Therefore, in the future, when studying multipresponse, scholars should can try to estimate more parameters with a few design points.

Acknowledgment. We would like to thank the referees and the journal editorial team for providing valuable advice that improved the quality of the original manuscript. This work was supported by the National Nature Sciences Foundation of China (12071096) and the Guangzhou University graduate innovative ability training funding program (2019GDJ C-D19).

\section{References}

[1] A.C. Atkinson, A.N. Donev and R.D. Tobias, Optimum Experiments Design, with $S A S$, Oxford, New York, 2007. 
[2] C.L. Atwood, Optimal and efficient designs of experiments, Ann. Math. Statist. 40 (5), 1570-1602, 1969.

[3] B. Ceranka and M. Graczyk, Regular A-optimal spring balance weighing designs with correlated errors, Hacet. J. Math. Stat. 44 (6), 1527-1535, 2015.

[4] S.I. Chang, Some properties of multiresponse D-optimal designs, J. Math. Anal. Appl. 184, 256-262, 1994.

[5] F. Chang, M.L. Huang, D.K.J. Lin and H. Yang, Optimal designs for dual response polynomial regression models, J. Statist. Plann. Inference 93 (1-2), 309-322, 2001.

[6] J.A. Cornell, Experiments with Mixtures, Designs, Models, and the Analysis of Mixture Data, 3rd ed, John Wiley and Sons, New York, 2002.

[7] N.R. Draper and W.G. Hunter, Design of experiments for parameter estimation in multiresponse situations, Biometrika 53 (3), 525-533, 1966.

[8] V.V. Federov, Theory of Optimal Experiments, Academic Press, New York, 1972.

[9] P. Goos, B. Jones and U. Syafitri, I-optimal design of mixture experiments, J. Amer. Statist. Assoc. 111 (514), 899-911, 2016.

[10] H.H. Hao and C.Q. Zhang, Multiresponse Scheffé mixture experiment optimal designs, in Chinese, Math. Prac. Theor. 48 (23), 183-188, 2018.

[11] W. Hassanein and N. Kilany, DE- and $E D P_{M^{-}}$compound optimality for the information and probability-based criteria, Hacet. J. Math. Stat. 48 (2), 580-591, 2019.

[12] L. Imhof, Optimum designs for a multiresponse regression model, J. Multivariate Anal. 72, 120-131, 2000.

[13] H. Jin and R.X. Yue, D-and A-optimal designs for mixture experiments with multiresponse models, Journal of Shanghai Normal University (Natural Sciences) 37 (2), 124-130, 2008.

[14] A.I. Khuri and J.A. Cornell, Response Surfaces: Designs and Analyses, Marcel Dekker, New York, 1987.

[15] J. Kiefer, General equivalence theory for optimal designs (approximate theory), Ann. Statist. 2 (5), 849-879, 1974.

[16] J. Kiefer, Optimum designs in regression problems, II, Ann. Math. Statist. 32 (1), 298-325, 1961.

[17] J. Kiefer and J. Wolfowitz, The equivalence of two extremum problems, Canad. J. Math. 12, 363-366, 1960.

[18] J. Kiefer and J. Wolfowitz, Optimum experimental designs, J. R. Stat. Soc. Ser. B. Stat. Methodol. 21 (2), 272-319, 1959.

[19] O. Krafft and M. Schaefer, D-optimal designs for a multivariate regression model, J. Multivariate Anal. 42, 130-140, 1992.

[20] P. Laake, On the optimal allocation of observations in experiments with mixtures, Scand. J. Stat. 2 (3), 153-157, 1975.

[21] C. Li and C.Q. Zhang, A-optimal designs for quadratic mixture canonical polynomials with spline, J. Statist. Plann. Inference 207, 1-9, 2020.

[22] G.H. Li and C.Q. Zhang, Random search algorithm for optimal mixture experimental design, Comm. Statist. Theory Methods 47 (6), 1413-1422, 2018.

[23] X. Liu and R.X. Yue, Design admissibility,invariance, and optimality in multipesponse linear models, Statist. Sinica 29, 2187-2203, 2019.

[24] X. Liu and R.X. Yue, A note on R-optimal designs for multiresponse models, Metrika 76, 483-493, 2013.

[25] X. Liu, R.X. Yue and W.K. Wong, D-optimal designs for multi-response linear mixed models, Metrika 82, 87-98, 2019.

[26] N.K. Mandal and M. Pal, Optimal designs for optimum mixtures in multiesponse experiments, Comm. Statist. Simulation Comput. 42 (5), 1104-1112, 2013.

[27] M. Nezhad, F. Saredorahi, M. Owlia and M. Zad. Design of economically and statistically optimal sampling plans, Hacet. J. Math. Stat. 47 (3), 685-708. 2018. 
[28] M. Pal and N.K. Mandal, Optimum designs for parameter estimation in a mixture experiment with two correlated responses, Comm. Statist. Simulation Comput. 46 (10), 7698-7709, 2017.

[29] F. Pukelsheim, Optimal Design of Experiments, John Wiley and Sons, New York, 2006.

[30] H. Scheffé, Simplex-centroid design for experiments with mixtures, J. R. Stat. Soc. Ser. B. Stat. Methodol. 25 (2), 235-263, 1963.

[31] H. Scheffé, Experiments with mixtures, J. R. Stat. Soc. Ser. B. Stat. Methodol. 20 (2), 344-360, 1958.

[32] W.F. Smith and J.A. Cornell, Biplot displays for looking at multiple response data in mixture experiments, Technometrics 35 (4), 337-350, 1993.

[33] M.C. Spruill and W.J. Studden, A Kiefer-Wolfowitz theorem in a stochastic process setting, Ann. Statist. 7 (6), 1329-1332, 1979.

[34] M.C. Wijesinha, Design of experiments for multiresponse models, PhD thesis, Department of Statistics, University of Florida, Gainesville, FL, 1984.

[35] W.K. Wong, Y. Yin and J. Zhou, Optimal designs for multi-response nonlinear regression models with several factors via semidefinite programming, J. Comput. Graph. Statist. 28 (1), 61-73, 2019.

[36] C.Q. Zhang and H. Peng, D-optimal designs for quadratic mixture canonical polynomials with spline, Statist. Probab. Lett. 82 (6), 1095-1101, 2012.

[37] Z.B. Zhu, G.H. Li and C.Q. Zhang, A-optimal designs for mixture central polynomial model with qualitative factors, Comm. Statist. Theory Methods 48 (10), 2345-2355, 2019 .

\section{Appendix}

We provide justifications for Theorems 3.3 and 3.5 using the same notation from the main text. In the following proof, for convenience, denote $\xi$ as the equal allocation design.

Proof of Theorem 3.3. (iii) deduces (ii), suppose the design $\xi^{*}$ satisfies:

$$
\sup _{x} \operatorname{Tr}\left(\Sigma^{-1} D\left(x, \xi^{*}, \Sigma\right)\right)=r .
$$

From Lemma 3.1, for any design we have

$$
\sup _{x} \operatorname{Tr}\left(\Sigma^{-1} D(x, \xi, \Sigma)\right) \geq r
$$

therefore

$$
\sup _{x} \operatorname{Tr}\left(\Sigma^{-1} D\left(x, \xi^{*}, \Sigma\right)\right)=\inf _{\xi}\left\{\sup _{x} \operatorname{Tr}\left(\Sigma^{-1} D(x, \xi, \Sigma)\right)\right\} .
$$

(i) deduces (iii), suppose the design $\xi^{*}$ satisfies:

$$
\operatorname{det}\left(M\left(\xi^{*}, \Sigma\right)\right)=\sup _{\xi} \operatorname{det}(M(\xi, \Sigma)) .
$$

Assume that the design $\xi^{*}$ does not satisfy (iii), so

$$
\sup _{x} \operatorname{Tr}\left(\Sigma^{-1} D\left(x, \xi^{*}, \Sigma\right)\right)>r
$$

so there is $x_{0} \in X$, st.

$$
\operatorname{Tr}\left(\Sigma^{-1} D\left(x_{0}, \xi^{*}, \Sigma\right)\right)>r .
$$

Let $\widetilde{\xi}=\xi^{*}+\xi_{x_{0}}$, where $\xi_{x_{0}}$ represents a single point with only one spectral point. So,

$$
M(\widetilde{\xi})=M\left(\xi^{*}\right)+M\left(\xi_{x_{0}}\right) .
$$

The convergence theorem of determinants of information matrix of iterative algorithm process according D-optimal design is as follows:

$$
\lim _{s \rightarrow \infty} \operatorname{det}\left(M\left(\xi_{s}\right)\right)=\operatorname{det}\left(M\left(\xi^{*}\right)\right)
$$


and from (A.1), we have $\operatorname{det}(M(\widetilde{\xi}))>\operatorname{det}\left(M\left(\xi^{*}\right)\right)$. This is against design $\xi^{*}$, satisfying (i).

(iii) deduces (i), suppose the design $\xi^{*}$ satisfies (iii):

$$
\sup _{x} \operatorname{Tr}\left(\Sigma^{-1} D\left(x, \xi^{*}, \Sigma\right)\right)=r .
$$

If the design $\xi^{*}$ does not satisfy (i), then there is design $\xi$ satisfying:

$$
\operatorname{det}\left(M\left(\xi^{*}\right)\right)<\operatorname{det}(M(\xi)) \text {. }
$$

Consider the design $\widetilde{\xi}=\xi^{*}+\xi$, so,

$$
M(\widetilde{\xi})=M\left(\xi^{*}\right)+M(\xi),
$$

therefore

$$
\operatorname{det}(M(\widetilde{\xi}))>\operatorname{det}(M(\xi)) \operatorname{det}\left(M\left(\xi^{*}\right)\right)>\operatorname{det}\left(M\left(\xi^{*}\right)\right)
$$

Given

$$
\operatorname{det}(M(\widetilde{\xi}))-\operatorname{det}\left(M\left(\xi^{*}\right)\right)>0,
$$

then

$$
\begin{aligned}
& \operatorname{det}(M(\widetilde{\xi}))-\operatorname{det}\left(M\left(\xi^{*}\right)\right) \\
= & \operatorname{Tr}\left(M^{-1}\left(\xi^{*}\right)\left(M(\xi)-M\left(\xi^{*}\right)\right)\right) \\
= & \operatorname{Tr}\left(M^{-1}\left(\xi^{*}\right) M(\xi)\right)-r \\
> & 0,
\end{aligned}
$$

this is

$$
\left(M^{-1}\left(\xi^{*}\right) M(\xi)\right)>r .
$$

Let

$$
\xi=\left(x_{1}, x_{2}, \ldots, x_{n}\right)
$$

so

$$
\begin{aligned}
\operatorname{Tr}\left(M^{-1}\left(\xi^{*}\right) M(\xi)\right) & =\operatorname{Tr}\left[M^{-1}\left(\xi^{*}\right) \sum f\left(x_{i}\right) f^{\mathrm{T}}\left(x_{i}\right)\right] \\
& =\sum \operatorname{Tr}\left[M^{-1}\left(\xi^{*}\right) f\left(x_{i}\right) f^{\mathrm{T}}\left(x_{i}\right)\right] \\
& =r f^{\mathrm{T}}\left(x_{i}\right) M^{-1}\left(\xi^{*}\right) f\left(x_{i}\right) \\
& =r D\left(x, \xi^{*}, \Sigma\right),
\end{aligned}
$$

because $D\left(x, \xi^{*}, \Sigma\right)>1$,

$$
\sup _{x} \operatorname{Tr}\left(\Sigma^{-1} D\left(x, \xi^{*}, \Sigma\right)\right),
$$

this is against design $\xi^{*}$, satisfying (iii).

(ii) deduces (iii), suppose design $\xi^{*}$ satisfies (ii):

$$
\operatorname{Tr}\left(\Sigma^{-1} D\left(x, \xi^{*}, \Sigma\right)\right)=\inf _{\xi} \sup _{x} \operatorname{Tr}\left(\Sigma^{-1} D(x, \xi), \Sigma\right) .
$$

If design $\xi^{*}$ is not satisfied (iii) then

$$
\sup _{x} \operatorname{Tr}\left(\Sigma^{-1} D\left(x, \xi^{*}, \Sigma\right)\right)>r .
$$

It has been proven that (i) is equivalent to (iii), so it does not satisfy (i), and with the definition of D-optimal design, D-optimal design must exist, that is

$$
\sup _{x} \operatorname{Tr}\left(\Sigma^{-1} D(x, \widetilde{\xi}, \Sigma)\right)=r,
$$


and because the design $\xi^{*}$ satisfies (ii), it is obtained by (A.3)

$$
\sup _{x} \operatorname{Tr}\left(\Sigma^{-1} D\left(x, \xi^{*}, \Sigma\right)\right) \leq r
$$

which conflicts (A.2).

Proof of Theorem 3.5. Let the information matrix of D-optimal design $\xi_{1}$ and $\xi_{2}$ be $M\left(\xi_{1}\right)$ and $M\left(\xi_{2}\right)$, respectively, and $M\left(\xi_{1}\right) \neq M\left(\xi_{2}\right)$.

Order $\xi=\xi_{1}+\xi_{2}$, then $M(\xi)=M\left(\xi_{1}\right)+M\left(\xi_{2}\right)$. From Lemma 3.2:

$$
\ln (\operatorname{det}(M(\xi)))>\ln \left(\operatorname{det}\left(M\left(\xi_{1}\right)\right)\right)+\ln \left(\operatorname{det}\left(M\left(\xi_{2}\right)\right)\right) .
$$

According to the definition of D-optimal design, we have

$$
\operatorname{det}\left(M\left(\xi_{1}\right)\right)=\operatorname{det}\left(M\left(\xi_{2}\right)\right) \geq \operatorname{det}(M(\xi)) .
$$

To make formula (A.4) and formula (A.5) not contradict each other, they must have

$$
\operatorname{det}\left(M\left(\xi_{1}\right)\right)=\operatorname{det}\left(M\left(\xi_{2}\right)\right)=\operatorname{det}(M(\xi)) \text {. }
$$

From the conclusions (i), (ii), (iii) and the formula (A.6) of Theorem 3.3, Theorem 3.5 is proved. 